\title{
Seleção de atrativos alimentares e toxicidade de inseticidas para o manejo da broca-pequena-do-tomateiro
}

\author{
Solange Maria de França(1), José Vargas de Oliveira(1), Marcelo Coutinho Picanço(2), \\ Ailton Pinheiro Lôbo(1), Ézio Marques da Silva ${ }^{(2)}$ e Pablo da Costa Gontijo(2)
}

\begin{abstract}
(1)Universidade Federal Rural de Pernambuco, Departamento de Agronomia/Entomologia, Avenida Dom Manoel de Medeiros, s/no, Dois Irmãos, CEP 52171-900 Recife, PE. E-mail: solangeufrpe@yahoo.com.br, vargasoliveira@uol.com.br, ailtonlobo@yahoo.com.br (2)Universidade Federal de Viçosa, Departamento de Biologia Animal, Avenida P.H. Rolfs, s/no, Campus Universitário, CEP 36570-000 Viçosa, MG. E-mail: picanco@ufv.br, eziomsilva@gmail.com, pablocgontijo@yahoo.com.br
\end{abstract}

Resumo - O objetivo deste trabalho foi avaliar a preferência alimentar, o limiar de ingestão e o efeito tóxico de inseticidas associados a atrativos, em adultos de Neoleucinodes elegantalis. Foram testados os atrativos: melado e mel a $10 \%$, extrato hexânico de frutos verdes de tomate a $0,4 \%$, sacarose a $5 \%$, suco de laranja e suco de uva a $30 \%$, vinagre de vinho tinto a $10 \%$ e proteína hidrolisada a $5 \%$. Com base no teste de preferência alimentar, foram selecionados os atrativos sacarose, melado, mel e suco de laranja, para determinar o limiar de concentração capaz de estimular a alimentação de adultos de $N$. elegantalis. Foi testado o efeito tóxico de inseticidas associados ao mel a $10 \%$. A sacarose e o mel apresentaram o melhor resultado em relação ao número de pousos e ao tempo de pouso e de alimentação de adultos de $N$. elegantalis. Os inseticidas não afetaram negativamente a atração pelo alimento dos adultos de N. elegantalis. Carbaril, cartape, deltametrina, fenpropatrina, indoxacarbe, lambda-cialotrina e lufenurom provocaram $100 \%$ de mortalidade em adultos (machos + fêmeas), após 24 horas de exposição, e mostraram-se promissores para o uso em iscas tóxicas.

Termos para indexação: Neoleucinodes elegantalis, controle comportamental, iscas tóxicas, limiar de resposta.

\section{Selection of attractive food sources and toxicity of insecticides in tomato fruit borer management}

\begin{abstract}
This work aimed at evaluating the food preference, the threshold of food intake and the toxic effect of insecticides associated with attractive food sources on adults of Neoleucinodes elegantalis. The following attractive food sources were tested: molasses and honey at $10 \%$, hexanic extract of green tomato fruits at $0.4 \%$, sucrose at $5 \%$, orange and grape juice at $30 \%$, red wine vinegar at $10 \%$, and hydrolyzed protein at $5 \%$ concentration. Based on the food preference test, sucrose, molasses, honey, and orange juice were selected to determine the threshold concentration capable of stimulating feeding in $N$. elegantalis adults. The toxic effect of insecticides added to honey at $10 \%$ was also tested. Sucrose and honey had the best results in terms of number of landings, landing time, and feeding time of adults of $N$. elegantalis. The insecticides did not affect negatively the attraction of $N$. elegantalis adults to the food sources. Carbaryl, cartap, deltamethrin, fenpropatrin, indoxacarb, lambda-cyhalothrin, and lufenuron caused $100 \%$ mortality of adults (males and females), after 24 hours of exposure, which suggests that they are promising for use in toxic baits.
\end{abstract}

Index terms: Neoleucinodes elegantalis, behavioral control, toxic baits, threshold response.

\section{Introdução}

A broca-pequena [Neoleucinodes elegantalis Guenée (Lepidoptera: Crambidae)] é uma das principais pragas da cultura do tomate no Brasil, Venezuela e Colômbia (Salas et al., 1991; Morais et al., 2007; Picanço et al., 2007). Em condições favoráveis ao crescimento populacional, infesta severamente os frutos, tornando-os impróprios para consumo e para o processamento industrial (Gravena \& Benvenga, 2003; Morais et al., 2007; Picanço et al., 2007).
Os estimulantes alimentares e os semioquímicos têm sido investigados como alternativas para o manejo comportamental de diferentes pragas. As substâncias químicas que indicam a presença do alimento são, em muitos casos, compostos secundários de plantas que estimulam as células quimiorreceptoras localizadas nas sensilas gustativas dos tarsos, antenas e partes do aparelho bucal dos insetos, e que induzem, entre outras ações, a alimentação e a oviposição (Nation, 2002).

Iscas atrativas com estimulantes alimentares têm sido utilizadas para a identificação e distribuição de 
espécies de insetos, certificação de uma região ou país quanto à ausência de determinada espécie-praga (área livre), e em programas de erradicação de espécies-praga e de manejo integrado (Nascimento et al., 2000). Para maior persistência dos fagoestimulantes e arrestantes nas iscas atrativas, utiliza-se o amido como veículo do estimulante alimentar (Arruda-Gatti \& Ventura, 2003), por exemplo, na combinação de farelos secos e inseticidas (Potts, 1999). Vários atrativos como açúcar mascavo, sacarose, proteína hidrolisada de milho, sucos de frutas e vinagre de vinho são utilizados em armadilhas, no monitoramento de insetos e, quando adicionados a inseticidas, são recomendados para o controle de pragas como as moscas-das-frutas (Nascimento et al., 2000; Gravena \& Benvenga, 2003).

Iscas tóxicas com spinosad e água, açúcar e outros atrativos foram efetivas no controle de moscas-das-frutas [Ceratitis capitata (Wied.) e Anastrepha fraterculus (Wied.)] (Raga \& Sato, 2005). No manejo da mosca-minadora (Liriomyza spp.), tem sido recomendado o uso do inseticida cartape associado a açúcar ou proteína hidrolisada (Guimarães et al., 2005).

$\mathrm{O}$ uso de iscas tóxicas, que contêm inseticidas comerciais registrados para a cultura do tomateiro, aplicadas no interior do cultivo e nas bordaduras, pode ser um método promissor para o controle de adultos de $N$. elegantalis. Esse método poderá contribuir para a redução do uso de inseticidas e gerar benefícios para o ambiente, pois não há necessidade de se fazerem aplicações em cobertura total.

O objetivo deste trabalho foi avaliar a preferência alimentar, o limiar de ingestão e o efeito tóxico de inseticidas associados a atrativo alimentar, no controle de adultos de $N$. elegantalis.

\section{Material e Métodos}

Os insetos foram criados conforme procedimento utilizado no Laboratório de Manejo Integrado de Pragas, do Departamento de Biologia Animal, da Universidade Federal de Viçosa, MG (Souza, 2001; Badji et al., 2003). A criação e os experimentos foram realizados a $26,2 \pm 1,7^{\circ} \mathrm{C}$, com umidade relativa de $75 \pm 0,7 \%$ e fotófase de 12 horas.

No estudo da atração alimentar, cada parcela experimental foi constituída de uma gaiola telada com organza $(45 \times 45 \times 45 \mathrm{~cm})$, na qual foram introduzidos cinco casais de $N$. elegantalis com quatro a cinco dias de idade, sem alimentação por 24 horas. Os tratamentos foram constituídos por chumaços de algodão $(0,33 \mathrm{~g}$ e $8 \mathrm{~cm}^{2}$ ) embebidos com $2 \mathrm{~mL}$ do atrativo alimentar. Os atrativos testados foram: melado e mel a $10 \%$, extrato hexânico de frutos verdes de tomate a $0,4 \%$, sacarose a $5 \%$, sucos de laranja e de uva a $30 \%$, vinagre de vinho tinto a $10 \%$ e proteína hidrolisada a $5 \%$. Esses tratamentos foram fixados com o auxílio de alfinetes no interior das gaiolas, na parte superior, dispostos de forma equidistante. Em cada repetição, foi realizada uma nova casualização da posição dos tratamentos, no interior das gaiolas.

Os experimentos foram realizados em sala, com fotófase de 12 horas. As avaliações foram visuais. Foram avaliadas as seguintes características: número de pousos de machos, fêmeas e adultos (machos + fêmeas) no atrativo alimentar; tempo de pouso de machos, fêmeas e adultos; número de vezes em que os machos, fêmeas e adultos se alimentaram; e tempo de alimentação de machos, fêmeas e adultos. Para a visualização dos adultos no interior das gaiolas, foram utilizadas lanternas revestidas com papel celofane de cor vermelha, para se evitar uma possível interferência da luz artificial no comportamento dos insetos (Wyatt, 1997). Os experimentos foram conduzidos em delineamento de blocos ao acaso, com oito tratamentos e quatro repetições. Os resultados obtidos foram submetidos à análise de variância, e as médias dos tratamentos foram comparadas pelo teste de agrupamento de Scott \& Knott (1974), a 5\% de probabilidade. As análises foram processadas por meio do SAEG 5.0 (Gomes, 1992).

Com base no teste de atração alimentar, foram selecionados os atrativos sacarose, melado, mel e suco de laranja, para se determinar o limiar de concentração capaz de estimular a alimentação de adultos de $N$. elegantalis. Foram testadas as seguintes concentrações: sacarose $(5,2,5,0,5,0,25,0,05,0,025,0,005$ e $0 \%)$; suco de laranja $(30,15,3,1,5,0,3,0,15,0,03$ e $0 \%)$; e mel e melado $(10,5,1,0,5,0,1,0,05,0,01$ e $0 \%)$. Os testes foram realizados em gaiolas de organza $(45 \times 45 \times 45 \mathrm{~cm})$, segundo o método descrito para o teste de atração alimentar. Os resultados obtidos foram submetidos à análise de variância, e foram estabelecidas correlações entre as características avaliadas que apresentaram significância $(\mathrm{p} \leq 0,10)$ e as concentrações de sacarose. 
Para avaliar a alteração na resposta de $N$. elegantalis, foram associados inseticidas à solução de mel. Esse atrativo foi usado em razão de seu desempenho. Cada parcela foi constituída de uma gaiola telada com organza $(45 \times 45 \times 45 \mathrm{~cm})$, na qual foram distribuídos 12 chumaços de algodão $\left(0,33 \mathrm{~g}\right.$ e $\left.8 \mathrm{~cm}^{2}\right)$ embebidos com $2 \mathrm{~mL}$ de solução de mel a $10 \%$ e com o inseticida. Como testemunha, foi utilizado apenas o atrativo. Os tratamentos, fixados com alfinetes na parte superior do interior das gaiolas, dispostos de forma equidistante, foram: testemunha, metomil (Lannate BR) $(100 \mathrm{~mL}$

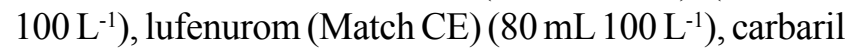
(Sevin $480 \mathrm{SC}$ ) $\left(225 \mathrm{~mL} 100 \mathrm{~L}^{-1}\right)$, deltametrina (Keshet $25 \mathrm{CE})\left(40 \mathrm{~mL} 100 \mathrm{~L}^{-1}\right)$, clorpirifós (Lorsban $480 \mathrm{BR}$ ) $\left(150 \mathrm{~mL} 100 \mathrm{~L}^{-1}\right)$, lambda-cialotrina (Karate Zeon $50 \mathrm{SC})\left(40 \mathrm{~mL} 100 \mathrm{~L}^{-1}\right)$, indoxacarbe (Rumo GDA)

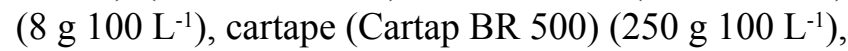

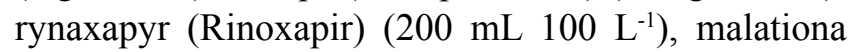
(Malathion $500 \mathrm{CE})\left(200 \mathrm{~mL} 100 \mathrm{~L}^{-1}\right)$ e fenpropatrina (Danimen $300 \mathrm{CE})\left(150 \mathrm{~mL} \mathrm{ha}^{-1}\right)$. Esses inseticidas são registrados para o controle de $N$. elegantalis em tomateiro. Em cada repetição, foi realizada uma nova casualização da posição dos tratamentos no interior das gaiolas.

Em cada gaiola, foram liberados cinco casais de $N$. elegantalis com quatro a cinco dias de idade, mantidos sem alimento por um período de 24 horas. Os experimentos foram realizados durante a escotófase, por seis horas ininterruptas, e verificados por meio de avaliações visuais. Foram avaliadas as mesmas variáveis utilizadas no experimento de atração alimentar. O delineamento experimental foi o de blocos ao acaso, com 12 tratamentos (11 inseticidas e testemunha) e seis repetições. Os resultados foram transformados em $(\mathrm{x}+0,5)^{0,5}$, para atender às pressuposições da ANOVA. As análises foram realizadas com o programa estatístico SAEG 5.0 (Gomes, 1992). Foi utilizada a análise de regressão, para avaliar o efeito dos inseticidas ao longo do tempo, na sobrevivência dos adultos de N. elegantalis.

A toxicidade desses inseticidas foi avaliada com o mesmo método do experimento anterior. Porém, os tratamentos (11 inseticidas e testemunha) foram individualizados em gaiolas teladas com organza $(45 \times 45 \times 45 \mathrm{~cm})$. Em cada gaiola, foram distribuídos 12 chumaços de algodão $\left(0,33 \mathrm{~g} \mathrm{e} 8 \mathrm{~cm}^{2}\right)$ embebidos com $2 \mathrm{~mL}$ de solução de mel a $10 \%$, e com o inseticida testado. A testemunha recebeu apenas solução de mel a $10 \%$. Os chumaços de algodão foram fixados com alfinete, na parte superior do interior da gaiola, e dispostos de forma equidistante. Cada inseticida foi testado na dosagem recomendada pelo fabricante. Em cada gaiola, foram utilizados três casais de $N$. elegantalis com quatro a cinco dias de idade, mantidos sem alimento por 24 horas. Após $0,5,1,2$, 12 e 24 horas, foi avaliada a mortalidade dos adultos. Os testes foram efetuados com 12 horas de escotófase. Para a visualização dos adultos mortos no interior das gaiolas, no escuro, foi utilizada uma lanterna revestida com papel celofane de cor vermelha (Wyatt, 1997). Os resultados obtidos foram transformados em $(\mathrm{x}+0,5)^{0,5}$, para atender as pressuposições da ANOVA. Em seguida, foi realizado o teste de agrupamento de Scott \& Knott (1974), com uso do programa estatístico SAEG 5.0 (Gomes, 1992).

\section{Resultados e Discussão}

O número de pousos dos machos de $N$. elegantalis foi semelhante em sacarose, mel, melado, suco de uva, suco de laranja e proteína hidrolisada, e diferiu em vinagre de vinho e extrato hexânico de frutos de tomate (Tabela 1). Entretanto, não houve diferença estatística entre os tratamentos, em relação ao tempo de pouso e de alimentação. Para as fêmeas, sacarose e mel desencadearam maior número de pousos, e o tempo de pouso foi mais expressivo em sacarose. Quanto ao número de vezes em que se alimentaram, a resposta foi significativa em sacarose, mel, suco de laranja e melado e, quanto ao tempo de alimentação, em sacarose, mel e suco de laranja. Sacarose, mel e melado foram os mais efetivos em favorecer o número de vezes em que os machos e fêmeas se alimentaram e o tempo de alimentação das fềmeas. Considerado o total de adultos (machos e fêmeas), o número de pousos teve destaque em sacarose, mel e melado; o tempo de pouso e a alimentação destacaram-se em sacarose, mel, melado, suco de uva e de laranja; e o tempo de alimentação destacou-se em sacarose e suco de laranja.

Os adultos de $N$. elegantalis responderam de modo diferente aos atrativos e parâmetros avaliados, com maior destaque para sacarose e mel. Apesar de as iscas atrativas com estimulantes alimentares tornarem o manejo de pragas por manipulação do comportamento uma ferramenta eficiente e contribuírem para reduzir a quantidade e aumentar a eficiência de agrotóxicos aplicados em sistemas de produção agrícolas (Potts, 
1999), poucos estudos sobre a sua utilização no manejo de lepidópteros foram desenvolvidos, com maior concentração de informações para dípteros como as moscas-das-frutas. Em relação a lepidópteros, armadilhas com soluções de melaço a $20 \%$ e de açúcar não refinado a 5,10 ou $20 \%$ capturaram maior número de adultos de Mocis latipes (Landolt, 1995). Entre vários atrativos, as iscas de banana foram as mais eficazes na coleta de adultos de Eudocima phalonia, praga de citros e de numerosas culturas comerciais de frutas no Pacífico, África, Ásia e Austrália (Reddy et al., 2007). Entretanto, em relação ao monitoramento de moscas-das-frutas, vários atrativos têm sido utilizados com níveis variados de eficiência, em diferentes armadilhas como hidrolisados de milho, melaço de cana e sucos de frutas (Mendonça et al., 2003; Tibola et al., 2005; Guerra et al., 2007).

Para a escolha de um atrativo adequado, alguns fatores devem ser considerados, como: custo; facilidade de aplicação; capacidade de atração de adultos, principalmente fêmeas; e tempo de pouso associado à alimentação, pois quanto maior o contato dos órgãos sensoriais dos insetos com a isca, maior será a eficácia do inseticida. No presente trabalho, a sacarose e o mel tiveram as melhores respostas de adultos de $N$. elegantalis quanto ao número de pousos associados à alimentação (Tabela 1). Por conseguinte, esses atrativos podem ser de grande utilidade no monitoramento e controle dessa praga.

A variação das concentrações de mel, melado e suco de laranja não alterou a resposta de $N$. elegantalis a esses atrativos, e não foi possível a determinação do limiar de ingestão. No entanto, foi observada relação entre as concentrações de sacarose e a sua atratividade. A resposta de $N$. elegantalis a esse atrativo foi crescente até a concentração de $1 \%$ e permaneceu constante a partir desta (Figura 1).

Os açúcares são substâncias com melhor potencial de alimentação e excelentes estimulantes alimentares, e a sacarose é o mais efetivo (Potts, 1999). Em alguns insetos, ocorre a expansão da probóscide, quando a concentração de açúcar exposta às suas quimiossensilas excede o valor do limiar de resposta. Essa resposta pode variar de acordo com o estágio de maturação e as condições fisiológicas - a exemplo da mosca-varejeira, Phormia regina (Amakawa, 2001) -, bem como com a aprendizagem do inseto, pois a extensão da probóscide está associada ao estímulo ao qual o inseto foi exposto,

Tabela 1. Efeito de diferentes atrativos no comportamento de pouso e alimentação de machos, fêmeas e adultos de Neoleucinodes elegantalis (temperatura de $26,2 \pm 1,7^{\circ} \mathrm{C}$, umidade relativa de $75 \pm 0,7 \%$ e fotófase de 12 horas).

\begin{tabular}{|c|c|c|c|c|}
\hline \multirow[t]{2}{*}{ Tratamento } & \multicolumn{2}{|c|}{ Pouso (média \pm erro-padrão) ${ }^{(1)}$} & \multicolumn{2}{|c|}{ Alimentação (médiaterro-padrão) ${ }^{(1)}$} \\
\hline & Número & Tempo (min) & Número & Tempo (min) \\
\hline & \multicolumn{4}{|c|}{ Machos } \\
\hline Sacarose $(5 \%)$ & $4,2 \pm 1,6 \mathrm{~A}$ & $82,0 \pm 62,0 \mathrm{~A}$ & $3,0 \pm 1,9 \mathrm{~A}$ & $67,2 \pm 53,3 \mathrm{~A}$ \\
\hline Melado $(10 \%)$ & $3,7 \pm 2,6 \mathrm{~A}$ & $45,2 \pm 53,5 \mathrm{~A}$ & $2,6 \pm 2,3 \mathrm{~A}$ & $22,5 \pm 37,4 \mathrm{~A}$ \\
\hline Suco de uva $(30 \%)$ & $2,5 \pm 1,3 \mathrm{~A}$ & $61,8 \pm 47,8 \mathrm{~A}$ & $0,8 \pm 0,7 \mathrm{~B}$ & $14,2 \pm 19,4 \mathrm{~A}$ \\
\hline Suco de laranja $(30 \%)$ & $1,7 \pm 0,8 \mathrm{~A}$ & $32,3 \pm 28,9 \mathrm{~A}$ & $0,9 \pm 0,4 \mathrm{~B}$ & $31,3 \pm 28,7 \mathrm{~A}$ \\
\hline Proteína hidrolisada (5\%) & $1,7 \pm 0,3 \mathrm{~A}$ & $18,5 \pm 17,1 \mathrm{~A}$ & $0,2 \pm 0,3 \mathrm{~B}$ & $0,5 \pm 0,8 \mathrm{~A}$ \\
\hline \multirow[t]{2}{*}{ Extrato de frutos verdes $(0,4 \%)$} & $0,0 \pm 0,0 \mathrm{~B}$ & $0,0 \pm 0,0 \mathrm{~A}$ & $0,0 \pm 0,0 \mathrm{~B}$ & $0,0 \pm 0,0 \mathrm{~A}$ \\
\hline & \multicolumn{4}{|c|}{ Fêmeas } \\
\hline Sacarose $(5 \%)$ & $4,4 \pm 0,9 \mathrm{~A}$ & $82,8 \pm 39,4 \mathrm{~A}$ & $1,9 \pm 0,4 \mathrm{~A}$ & $19,6 \pm 11,4 \mathrm{~A}$ \\
\hline $\operatorname{Mel}(10 \%)$ & $2,8 \pm 0,9 \mathrm{~A}$ & $24,0 \pm 21,3 \mathrm{~A}$ & $2,3 \pm 0,9 \mathrm{~A}$ & $19,0 \pm 20,3 \mathrm{~A}$ \\
\hline Suco de laranja (30\%) & $1,7 \pm 0,8 \mathrm{~B}$ & $31,4 \pm 26,4 \mathrm{~A}$ & $1,1 \pm 0,5 \mathrm{~A}$ & $27,1 \pm 26,3 \mathrm{~A}$ \\
\hline Melado $(10 \%)$ & $1,3 \pm 0,7 \mathrm{~B}$ & $4,1 \pm 2,2 \mathrm{~B}$ & $1,0 \pm 0,8 \mathrm{~A}$ & $2,9 \pm 2,5 \mathrm{~B}$ \\
\hline Suco de uva $(30 \%)$ & $1,1 \pm 0,6 \mathrm{~B}$ & $16,8 \pm 18,6 \mathrm{~A}$ & $0,6 \pm 0,5 \mathrm{~B}$ & $2,5 \pm 2,1 \mathrm{~B}$ \\
\hline Proteína hidrolisada (5\%) & $0,8 \pm 0,7 \mathrm{~B}$ & $0,8 \pm 0,6 \mathrm{~B}$ & $0,2 \pm 0,3 \mathrm{~B}$ & $0,4 \pm 0,5 \mathrm{~B}$ \\
\hline \multirow[t]{2}{*}{ Extrato de frutos verdes $(0,4 \%)$} & $0,0 \pm 0,0 \mathrm{~B}$ & $0,0 \pm 0,0 \mathrm{~B}$ & $0,0 \pm 0,0 \mathrm{~B}$ & $0,0 \pm 0,0 \mathrm{~B}$ \\
\hline & \multicolumn{4}{|c|}{ Adultos } \\
\hline Sacarose $(5 \%)$ & $8,9 \pm 0,9 \mathrm{~A}$ & $203,3 \pm 30,99 \mathrm{~A}$ & $5,3 \pm 1,5 \mathrm{~A}$ & $97,4 \pm 47,76 \mathrm{~A}$ \\
\hline $\operatorname{Mel}(10 \%)$ & $6,8 \pm 1,89 \mathrm{~A}$ & $64,4 \pm 35,19 \mathrm{~A}$ & $3,9 \pm 1,5 \mathrm{~A}$ & $35,0 \pm 23,81 \mathrm{~B}$ \\
\hline Melado $(10 \%)$ & $5,4 \pm 2,98 \mathrm{~A}$ & $54,3 \pm 55,03 \mathrm{~A}$ & $3,9 \pm 2,78 \mathrm{~A}$ & $28,7 \pm 39,3 \mathrm{~B}$ \\
\hline Suco de uva $(30 \%)$ & $4,0 \pm 1,11 \mathrm{~B}$ & $97,5 \pm 44,6 \mathrm{~A}$ & $1,9 \pm 0,5 \mathrm{~A}$ & $23,0 \pm 17,8 \mathrm{~B}$ \\
\hline Suco de laranja (30\%) & $3,8 \pm 1,15 \mathrm{~B}$ & $94,1 \pm 14,98 \mathrm{~A}$ & $2,2 \pm 0,5 \mathrm{~A}$ & $87,5 \pm 17,69 \mathrm{~A}$ \\
\hline Proteína hidrolisada (5\%) & $2,7 \pm 0,5 \mathrm{~B}$ & $20,3 \pm 16,89 \mathrm{~B}$ & $0,4 \pm 0,5 \mathrm{~B}$ & $0,7 \pm 1,3 \mathrm{C}$ \\
\hline Vinagre de vinho $(10 \%)$ & $1,1 \pm 0,5 \mathrm{C}$ & $11,1 \pm 25,3 \mathrm{~B}$ & $0,2 \pm 0,3 \mathrm{~B}$ & $0,0 \pm 0,0 \mathrm{C}$ \\
\hline Extrato de frutos verdes $(0,4 \%)$ & $0,0 \pm 0,0 \mathrm{C}$ & $0,0 \pm 0,0 \mathrm{~B}$ & $0,0 \pm 0,0 \mathrm{~B}$ & $0,0 \pm 0,0 \mathrm{C}$ \\
\hline
\end{tabular}

${ }^{(1)}$ Médias seguidas de letras iguais, nas colunas, não diferem entre si pelo teste de Scott-Knott, a 5\% de probabilidade. 
como no caso de abelhas (Scheiner, 2004). Esse comportamento também foi observado em algumas espécies de mariposas, como Spodoptera littoralis que foi capaz de associar um odor neutro (óleo de geraniol) à recompensa à sacarose (Fan et al., 1997).

A atratividade dos inseticidas, quando associados à solução de mel a $10 \%$, não diferiu estatisticamente quanto: ao número de pousos de machos, fềmeas e adultos no atrativo alimentar; ao tempo de pouso de fêmeas e adultos; ao número de vezes em que os machos, fêmeas e adultos se alimentaram; e ao tempo de alimentação para machos, fêmeas e adultos, em relação à testemunha (solução de mel a $10 \%$ ). Não houve alteração na resposta dos adultos ao atrativo, porém a presença do
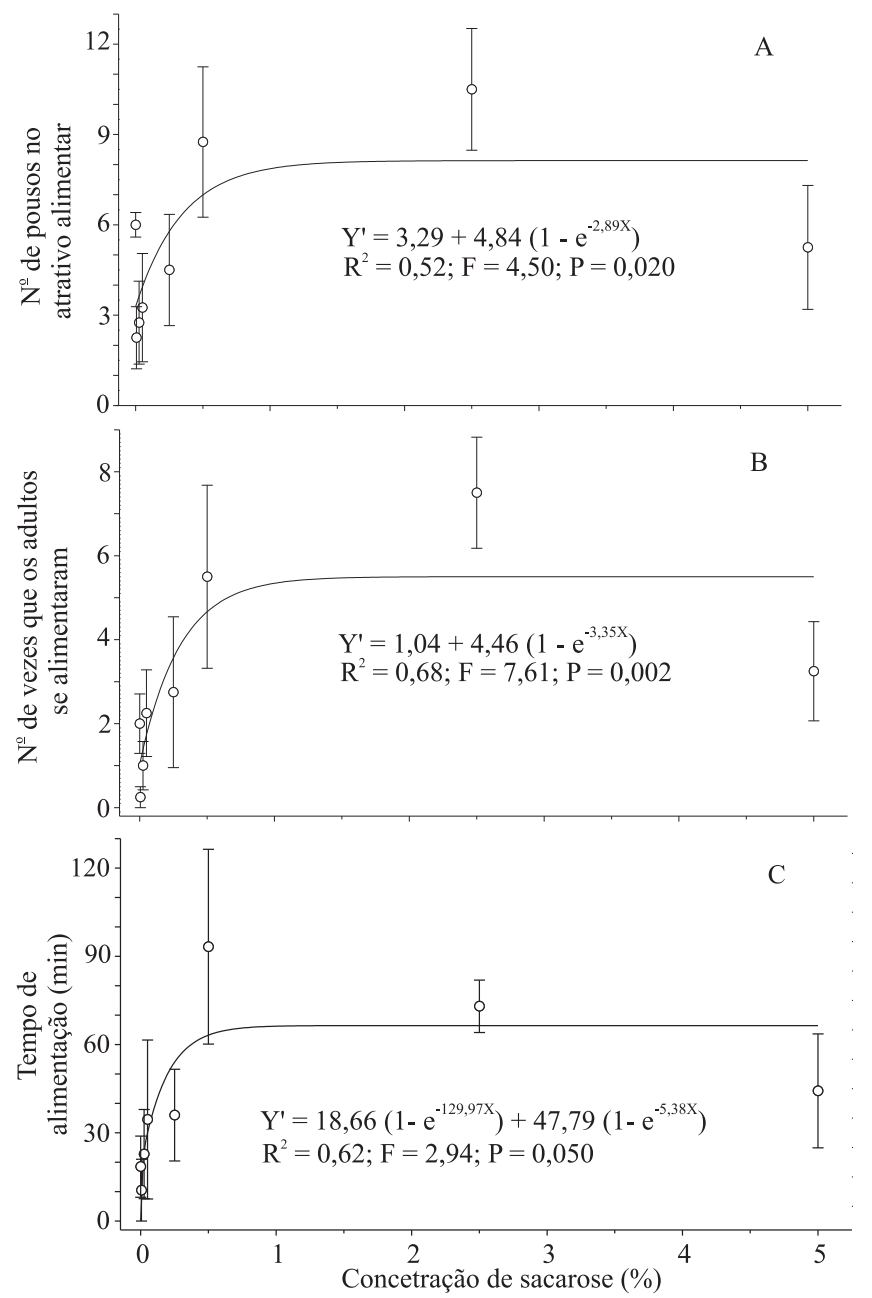

Figura 1. Número de pousos no atrativo alimentar (A); número de vezes (B) e tempo de alimentação (C) dos adultos de Neoleucinodes elegantalis, em razão da concentração de sacarose (temperatura de $26,2 \pm 1,7^{\circ} \mathrm{C}$, umidade relativa de $75 \pm 0,7 \%$ e escotófase de 12 horas). inseticida afetou significativamente a sobrevivência de fêmeas, machos e adultos de $N$. elegantalis, ao longo do tempo (Figura 2). Assim, fica demonstrado o potencial dos inseticidas testados para o manejo dessa praga com iscas tóxicas em tomateiro, pois nenhum deles apresentou efeito repelente, o que poderia interferir significativamente na resposta do inseto ao atrativo alimentar.

A mortalidade de N. elegantalis, causada pelos inseticidas, aumentou diretamente com os períodos de avaliação para machos, fềmeas e adultos (Tabela 2). Deltametrina provocou mortalidade de $66,7 \%$ após meia hora de observação. Após duas horas, os melhores resultados foram obtidos com deltametrina, lufenurom e carbaril; com 12 horas de observação, carbaril, cartape, deltametrina, indoxacarbe e lufenurom proporcionaram os melhores resultados; e, após 24 horas, destacaram-se carbaril, cartape, deltametrina,
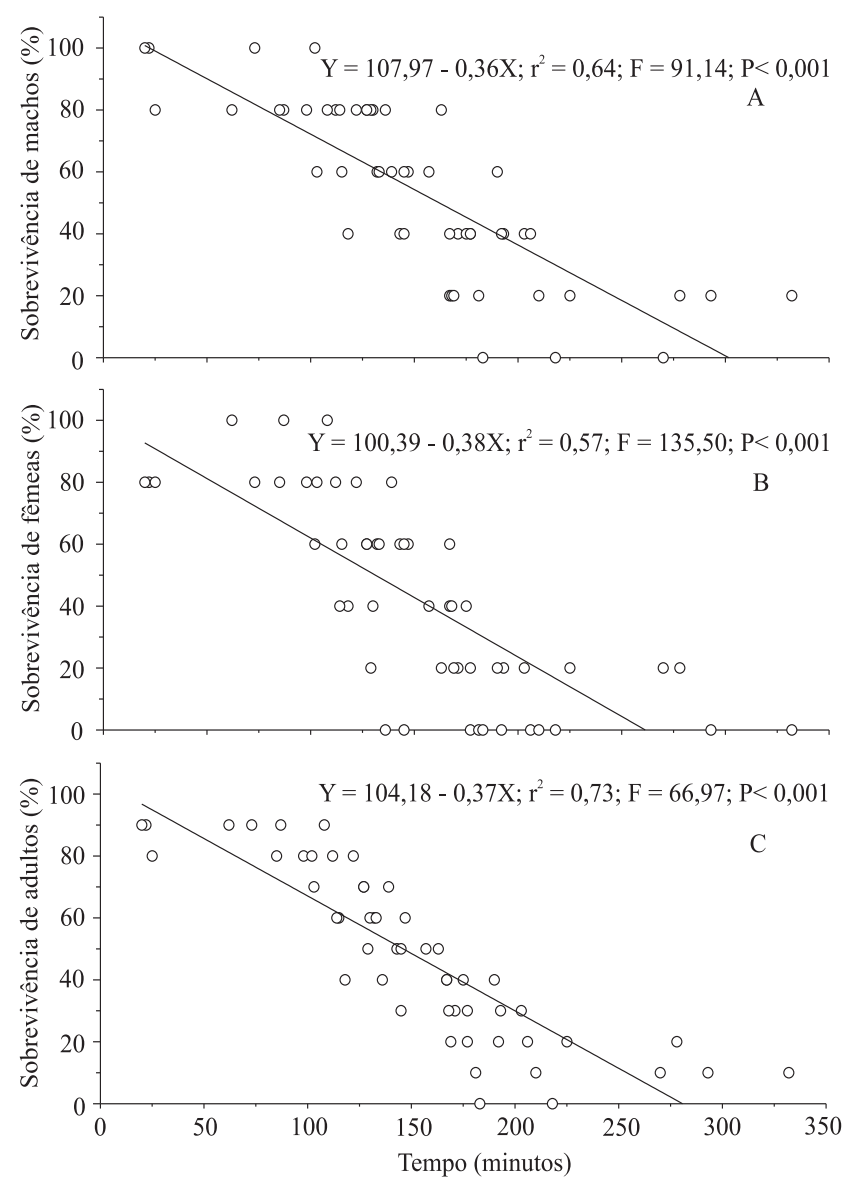

Figura 2. Sobrevivência de machos (A), fêmeas (B) e adultos (machos + fêmeas) (C) de Neoleucinodes elegantalis submetidos a 11 inseticidas, em função do tempo (min) (temperatura de $26,2 \pm 1,7^{\circ} \mathrm{C}$, umidade relativa de $75 \pm 0,7 \%$ e escotófase de 12 horas). 
Tabela 2. Mortalidade \pm erro-padrão de machos, fêmeas e adultos totais, em consequência de 11 inseticidas fornecidos em associação com solução de mel a $10 \%$ como atrativo alimentar (temperatura de $26,2 \pm 1,7^{\circ} \mathrm{C}$, umidade relativa de $75 \pm 0,7 \%$ e escotófase de 12 horas) $)^{(1)}$.

\begin{tabular}{|c|c|c|c|c|c|}
\hline \multirow[t]{2}{*}{ Tratamento } & \multicolumn{5}{|c|}{ Tempo (horas) } \\
\hline & 0,5 & 1 & 2 & 12 & 24 \\
\hline & \multicolumn{5}{|c|}{ Macho } \\
\hline Carbaril & $0,0 \pm 0,0 \mathrm{bB}$ & $0,0 \pm 0,0 \mathrm{bC}$ & $88,8 \pm 11,1 \mathrm{Aa}$ & $100,0 \pm 0,0 \mathrm{aA}$ & $100,0 \pm 0,0 \mathrm{aA}$ \\
\hline Cartape & $0,0 \pm 0,0 \mathrm{bB}$ & $0,0 \pm 0,0 \mathrm{bC}$ & $0,0 \pm 0,0 \mathrm{bC}$ & $100,0 \pm 0,0 \mathrm{aA}$ & $100,0 \pm 0,0 \mathrm{aA}$ \\
\hline Deltametrina & $66,7 \pm 19,3 \mathrm{aA}$ & $88,8 \pm 11,1 \mathrm{aA}$ & $100,0 \pm 0,0 \mathrm{aA}$ & $100,0 \pm 0,0 \mathrm{aA}$ & $100,0 \pm 0,0 \mathrm{aA}$ \\
\hline Fenpropatrina & $0,0 \pm 0,0 \mathrm{bB}$ & $0,0 \pm 0,0 \mathrm{bC}$ & $0,0 \pm 0,0 \mathrm{bC}$ & $77,7 \pm 22,2 \mathrm{aA}$ & $100,0 \pm 0,0 \mathrm{aA}$ \\
\hline Indoxacarbe & $0,0 \pm 0,0 \mathrm{cB}$ & $0,0 \pm 0,0 \mathrm{cC}$ & $44,4 \pm 11,1 \mathrm{bB}$ & $100,0 \pm 0,0 \mathrm{aA}$ & $100,0 \pm 0,0 \mathrm{aA}$ \\
\hline Lambda-cialotrina & $0,0 \pm 0,0 \mathrm{cB}$ & $33,3 \pm 19,2 \mathrm{bB}$ & $55,5 \pm 11,1 \mathrm{aB}$ & $77,7 \pm 22,2 \mathrm{aA}$ & $100,0 \pm 0,0 \mathrm{aA}$ \\
\hline Malationa & $0,0 \pm 0,0 \mathrm{bB}$ & $0,0 \pm 0,0 \mathrm{bC}$ & $0,0 \pm 0,0 \mathrm{bC}$ & $55,5 \pm 22,2 \mathrm{aA}$ & $55,5 \pm 22,2 \mathrm{aA}$ \\
\hline Clorpirifós & $0,0 \pm 0,0 \mathrm{bB}$ & $0,0 \pm 0,0 \mathrm{bC}$ & $0,0 \pm 0,0 \mathrm{bC}$ & $11,1 \pm 11,1 \mathrm{bB}$ & $33,3 \pm 19,2 \mathrm{aB}$ \\
\hline Rynaxapyr & $0,0 \pm 0,0 \mathrm{bB}$ & $0,0 \pm 0,0 \mathrm{bC}$ & $0,0 \pm 0,0 \mathrm{bC}$ & $22,2 \pm 11,1 \mathrm{aB}$ & $33,3 \pm 19,2 \mathrm{aB}$ \\
\hline Metomil & $11,1 \pm 11,1 \mathrm{aB}$ & $11,1 \pm 11,1 \mathrm{aC}$ & $11,1 \pm 11,1 \mathrm{aC}$ & $11,1 \pm 11,1 \mathrm{aB}$ & $22,2 \pm 11,1 \mathrm{aB}$ \\
\hline \multirow[t]{2}{*}{ Testemunha } & $0,0 \pm 0,0 \mathrm{aB}$ & $0,0 \pm 0,0 \mathrm{aC}$ & $0,0 \pm 0,0 \mathrm{aC}$ & $11,1 \pm 11,1 \mathrm{aB}$ & $11,1 \pm 11,1 \mathrm{aB}$ \\
\hline & \multicolumn{5}{|c|}{ Fêmea } \\
\hline Carbaril & $0,0 \pm 0,0 \mathrm{bB}$ & $0,0 \pm 0,0 \mathrm{bC}$ & $88,9 \pm 11,1 \mathrm{aA}$ & $100,0 \pm 0,0 \mathrm{aA}$ & $100,0 \pm 0,0 \mathrm{aA}$ \\
\hline Cartape & $0,0 \pm 0,0 \mathrm{bB}$ & $11,1 \pm 11,1 \mathrm{bC}$ & $0,0 \pm 0,0 \mathrm{bC}$ & $100,0 \pm 0,0 \mathrm{aA}$ & $100,0 \pm 0,0 \mathrm{aA}$ \\
\hline Fenpropatrina & $0,0 \pm 0,0 \mathrm{cB}$ & $0,0 \pm 0,0 \mathrm{cC}$ & $0,0 \pm 0,0 \mathrm{cC}$ & $66,6 \pm 33,33 \mathrm{bA}$ & $100,0 \pm 0,0 \mathrm{aA}$ \\
\hline Indoxacarbe & $0,0 \pm 0,0 \mathrm{cB}$ & $0,0 \pm 0,0 \mathrm{cC}$ & $44,4 \pm 22,2 \mathrm{bB}$ & $100,0 \pm 0,0 \mathrm{aA}$ & $100,0 \pm 0,0 \mathrm{aA}$ \\
\hline Lufenurom & $0,0 \pm 0,0 \mathrm{bB}$ & $11,1 \pm 11,1 \mathrm{bC}$ & $100,0 \pm 0,0 \mathrm{aA}$ & $100,0 \pm 0,0 \mathrm{aA}$ & $100,0 \pm 0,0 \mathrm{aA}$ \\
\hline Lambda-cialotrina & $0,0 \pm 0,0 \mathrm{cB}$ & $33,3 \pm 19,2 \mathrm{bB}$ & $66,7 \pm 19,2 \mathrm{aA}$ & $88,8 \pm 11,1 \mathrm{aA}$ & $88,8 \pm 11,1 \mathrm{aA}$ \\
\hline Malationa & $0,0 \pm 0,0 \mathrm{bB}$ & $0,0 \pm 0,0 \mathrm{bC}$ & $0,0 \pm 0,0 \mathrm{bC}$ & $77,7 \pm 11,1 \mathrm{aA}$ & $77,7 \pm 11,1 \mathrm{aA}$ \\
\hline Rynaxapyr & $0,0 \pm 0,0 \mathrm{cB}$ & $0,0 \pm 0,0 \mathrm{cC}$ & $0,0 \pm 0,0 \mathrm{cC}$ & $22,2 \pm 11,1 \mathrm{bB}$ & $55,5 \pm 11,1 \mathrm{aA}$ \\
\hline Metomil & $11,1 \pm 11,1 \mathrm{aB}$ & $11,1 \pm 11,1 \mathrm{aC}$ & $11,1 \pm 11,1 \mathrm{aC}$ & $33,3 \pm 19,2 \mathrm{aB}$ & $33,3 \pm 19,2 \mathrm{aB}$ \\
\hline Clorpirifós & $0,0 \pm 0,0 \mathrm{aB}$ & $0,0 \pm 0,0 \mathrm{aC}$ & $0,0 \pm 0,0 \mathrm{aC}$ & $22,2 \pm 22,2 \mathrm{aB}$ & $22,2 \pm 22,2 \mathrm{aB}$ \\
\hline \multirow[t]{2}{*}{ Testemunha } & $0,0 \pm 0,0 \mathrm{aB}$ & $0,0 \pm 0,0 \mathrm{aC}$ & $0,0 \pm 0,0 \mathrm{aC}$ & $0,0 \pm 0,0 \mathrm{aC}$ & $0,0 \pm 0,0 \mathrm{aC}$ \\
\hline & \multicolumn{5}{|c|}{ Total de adultos } \\
\hline Carbaril & $0,0 \pm 0,0 \mathrm{bB}$ & $0,0 \pm 0,0 \mathrm{bC}$ & $88,9 \pm 11,1 \mathrm{aA}$ & $100,0 \pm 0,0 \mathrm{aA}$ & $100,0 \pm 0,0 \mathrm{aA}$ \\
\hline Cartape & $0,0 \pm 0,0 \mathrm{bB}$ & $0,0 \pm 0,0 \mathrm{bC}$ & $0,0 \pm 0,0 \mathrm{bC}$ & $100,0 \pm 0,0 \mathrm{aA}$ & $100,0 \pm 0,0 \mathrm{aA}$ \\
\hline Deltametrina & $66,7 \pm 16,7 \mathrm{aA}$ & $83,3 \pm 16,7 \mathrm{aA}$ & $100,0 \pm 0,0 \mathrm{aA}$ & $100,0 \pm 0,0 \mathrm{aA}$ & $100,0 \pm 0,0 \mathrm{aA}$ \\
\hline Fenpropatrina & $0,0 \pm 0,0 \mathrm{bB}$ & $0,0 \pm 0,0 \mathrm{bC}$ & $0,0 \pm 0,0 \mathrm{bC}$ & $72,2 \pm 14,7 \mathrm{aA}$ & $100,0 \pm 0,0 \mathrm{aA}$ \\
\hline Indoxacarbe & $0,0 \pm 0,0 \mathrm{cB}$ & $5,5 \pm 5,6 \mathrm{cC}$ & $44,4 \pm 14,7 \mathrm{bB}$ & $100,0 \pm 0,0 \mathrm{aA}$ & $100,0 \pm 0,0 \mathrm{aA}$ \\
\hline Lufenurom & $0,0 \pm 0,0 \mathrm{bB}$ & $11,1 \pm 11,1 \mathrm{bC}$ & $100,0 \pm 0,0 \mathrm{aA}$ & $100,0 \pm 0,0 \mathrm{aA}$ & $100,0 \pm 0,0 \mathrm{aA}$ \\
\hline Lambda-cialotrina & $0,0 \pm 0,0 \mathrm{cB}$ & $33,3 \pm 19,2 \mathrm{bB}$ & $61,1 \pm 14,7 \mathrm{aB}$ & $83,3 \pm 16,7 \mathrm{aA}$ & $94,4 \pm 5,6 \mathrm{aA}$ \\
\hline Clorpirifós & $0,0 \pm 0,0 \mathrm{bB}$ & $0,0 \pm 0,0 \mathrm{bC}$ & $0,0 \pm 0,0 \mathrm{bC}$ & $16,6 \pm 16,7 \mathrm{aB}$ & $27,7 \pm 14,7 \mathrm{aB}$ \\
\hline Metomil & $11,1 \pm 11,1 \mathrm{aB}$ & $11,1 \pm 11,1 \mathrm{aC}$ & $11,1 \pm 11,1 \mathrm{aC}$ & $22,2 \pm 14,7 \mathrm{aB}$ & $27,7 \pm 14,7 \mathrm{aB}$ \\
\hline Testemunha & $0,0 \pm 0,0 \mathrm{aB}$ & $0,0 \pm 0,0 \mathrm{aC}$ & $0,0 \pm 0,0 \mathrm{aC}$ & $5,5 \pm 5,6 \mathrm{aB}$ & $5,5 \pm 5,6 \mathrm{aC}$ \\
\hline
\end{tabular}

${ }^{(1)}$ Médias seguidas por letras iguais, maiúsculas nas colunas e minúsculas nas linhas, não diferem entre si pelo teste de Scott-Knott, a $5 \%$ de probabilidade.

fenpropatrina, indoxacarbe, lambda-cialotrina e lufenuron.

Em relação a outros lepidópteros, Gallo et al. (2002) indicaram para o controle de mariposas em algodoeiro e soja: aplicação da mistura de melaço (1 L), água $(10 \mathrm{~L})$ e inseticida metomil a 21,5\% (30 mL) em $5 \mathrm{~L}$, por $5 \mathrm{~m}$ de linha de plantas, em faixas com afastamento de $50 \mathrm{~m}$. Iscas do inseticida cartape, nas doses de 500 e $750 \mathrm{~g}$ i.a. ha $^{-1}$ adicionadas a $0,5 \%$ de açúcar, foram efetivas no controle de adultos da lagarta rosada do algodoeiro, Pectinophora gossypiella (Saunders) (Papa et al., 2003).

No manejo de $N$. elegantalis, as aplicações de iscas tóxicas podem contribuir para a redução do uso de inseticidas, bem como beneficiar o ambiente e inimigos naturais, em comparação ao sistema convencional de aplicação de inseticidas. Além disso, há redução dos custos de produção e dos resíduos no tomate comercializado.

\section{Conclusões}

1. Os atrativos sacarose e mel são os mais eficientes em relação ao número de pousos e alimentação, tanto para machos quanto para fêmeas de Neoleucinodes elegantalis.

2. O limiar de ingestão da sacarose por N. elegantalis é de $1 \%$. 
3. Os inseticidas metomil, lufenurom, carbaril, deltametrina, clorpirifós, lambda-cialotrina, indoxacarbe, cartape, rynaxapyr, malationa e fenpropatrina não alteram a atratividade, quando associados ao mel.

4. Carbaril, cartape, deltametrina, fenpropatrina, indoxacarbe, lambda-cialotrina e lufenurom, quando associados ao mel, são promissores para o manejo de $N$. elegantalis em iscas tóxicas.

\section{Agradecimentos}

À Coordenação de Aperfeiçoamento de Pessoal de Nível Superior, ao Conselho Nacional de Desenvolvimento Científico e Tecnológico, à Fundação de Amparo à Pesquisa do Estado de Minas Gerais e ao Programa de Cooperação Acadêmica da Capes entre as Universidades Federal Rural de Pernambuco e Federal de Viçosa, pelas bolsas e recursos concedidos; aos colegas do Laboratório de Manejo Integrado de Pragas, da Universidade Federal de Viçosa, pela ajuda dispensada.

\section{Referências}

AMAKAWA, T. Effects of age and blood sugar levels on the proboscis extension of the blow fly Phormia regina. Journal of Insect Physiology, v.47, p.195-203, 2001.

ARRUDA-GATTI, I.C.; VENTURA, M.U. Iscas contendo cucurbitacinas para o manejo de Diabrotica spp. Semina. Ciências Agrárias, v.24, p.331-336, 2003.

BADJI, C.A.; EIRAS, A.E.; CABRERA, A.; JAFFE, K. Avaliação do feromônio sexual de Neoleucinodes elegantalis Guenée (Lepidoptera: Crambidae). Neotropical Entomology, v.32, p.221229, 2003.

FAN, R.J.; ANDERSON, P.; HANSSON, B.S. Behavioural analysis of olfactory conditioning in the moth Spodoptera littoralis (Boisd.) (Lepidoptera: Noctuidae). Journal of Experimental Biology, v.200, p.2969-2976, 1997.

GALLO, D.; NAKANO, O.; SILVEIRA NETO, S.; CARVALHO, R.P.L.; BATISTA, G.C. de; BERTI FILHO, E.; PARRA, J.R.P.; ZUCCHI, R.A.; ALVES, S.B.; VENDRAMIN, J.D.; MARCHINI, L.C.; LOPES, J.R.S.; OMOTO, C. Entomologia agrícola. Piracicaba: Fealq, 2002.920p.

GOMES, J.M. SAEG: sistema de análises estatísticas e genéticas. Versão 5.0. Viçosa: UFV, 1992. 100p.

GRAVENA, S.; BENVENGA, S.R. Manual prático para manejo ecológico de pragas do tomate. Jaboticabal: Gravena-ManEcol, 2003. 144p.

GUERRA, D.S.; MARODIN, G.A.B.; ZANINI, C.L.D.; ARGENTA, F.; GRASELLI, V.; NUNES, J.L. da S. Utilização de pesticidas na produção de pêssegos 'Marli', nos sistemas de produção integrada e convencional. Revista Brasileira de Fruticultura, v.29, p.91-95, 2007.

GUIMARÃES, J.A.; AZEVEDO, F.R. de; BRAGA SOBRINHO, R.; MESQUITA, A.L.M. Recomendações para o manejo das principais pragas do meloeiro na região do Semi-Árido Nordestino. Fortaleza: Embrapa Agroindústria Tropical, 2005. 11p. (Embrapa Agroindústria Tropical. Circular Técnica, 24).

LANDOLT, P.J. Attraction of Mocis latipes (Lepidoptera: Noctuidae) to sweet baits in traps. Florida Entomologist, v.78, p.523-530, 1995.

MENDONÇA, M. da C.; NASCIMENTO, A.S. do; MELO, A.S. de. Eficiência de atratividade da isca fotoativa para moscas-das-frutas (Diptera: Tephritidae). Revista Ciência Agronômica, v.34, p.147-152, 2003.

MORAIS, E.G.F. de; PICANÇO, M.C.; SENA, M.E. de; BACCI, L.; SILVA, G.A.; CAMPOS, M.R. de. Identificação das principais pragas de hortaliças no Brasil. In: ZAMBOLIM, L.; LOPES, C.A.; PICANÇO, M.C.; COSTA, H. (Ed.). Manejo integrado de doenças e pragas: hortaliças. Viçosa: UFV, 2007. p.199-232.

NASCIMENTO, A.S.; CARVALHO, R.S.; MALAVASI, A. Monitoramento populacional. In: MALAVASI, A.; ZUCCHI, R.A. (Ed.). Moscas-das-frutas de importância econômica no Brasil: conhecimento básico e aplicado. Ribeirão Preto: Holos, 2000. p.109-112.

NATION, J.L. Nutrition. In: NATION, J.L. (Ed.). Insect physiology and biochemistry. Boca Raton: CRC Press, 2002. p.65-87.

PAPA, G.; CELOTO, F.J.; TAKAO, W.; PRADO, E.P. Efeito da isca cartap + açúcar sobre adultos da lagarta rosada, Pectinophora gossypiella (Saunders, 1844) (Lepidoptera: Gelechiidae). In: CONGRESSO BRASILEIRO DE ALGODÃO, 4., 2003, Goiânia. Algodão: um mercado em evolução: anais. Campina Grande: Embrapa Algodão; Goiânia: Fundação GO, 2003. 1 CD-ROM. (Embrapa Algodão. Documentos, 118).

PICANÇO, M.C.; BACCI, L.; SILVA, E.M.; MORAIS, E.G.F.; SILVA, G.A.; SILVA, N.R. Manejo integrado das pragas do tomateiro no Brasil. In: SILVA, D.J.H.; VALE, F.X.R. (Ed.). Tomate: tecnologia de produção. Viçosa: UFV, 2007. p.199-232.

POTTS, L. Feeding stimulants and semiochemicals as pest management tools. 1999. Disponível em: <http://www.colostate. edu/Depts/Entomology/courses/en507/papers_1999/potts.htm>. Acesso em: 27 dez. 2007.

RAGA, A.; SATO, M.E. Effect of spinosad bait against Ceratitis capitata (Wied.) and Anastrepha fraterculus (Wied.) (Diptera: Tephritidae) in laboratory. Neotropical Entomology, v.34, p.815-822, 2005.

REDDY, G.V.P.; CRUZ, Z.T.; MUNIAPPAN, R. Attraction of fruit-piercing moth Eudocima phalonia (Lepidoptera: Noctuidae) to different fruit baits. Crop Protection, v.26, p.664-667, 2007.

SALAS, J.; ALVAREZ, C.; PARRA, A. Contribución al conocimiento de la ecología del perforador del fruto del tomate Neoleucinodes elegantalis Guenée (Lepidoptera: Pyraustidae). Agronomía Tropical, v.41, p.275-284, 1991. 
SCHEINER, R. Responsiveness to sucrose and habituation of the proboscis extension response in honey bees. Journal of Comparative Physiology A, v.190, p.727-733, 2004.

SCOTT, A.J.; KNOTT, M. A cluster analysis method for grouping means in the analysis of variance. Biometrics, v.30, p.507-512, 1974.

SOUZA, C.L.M. Influência de aleloquímicos na interação tritrófica entre Lycopersicon spp. Miller (Solanales: Solanaceae), Trichogramma pretiosum Riley (Hymenoptera: Trichogrammatidae) e Neoleucinodes elegantalis (Guenée)
(Lepidoptera: Crambidae), Campos dos Goitacazes. 2001. 124p. Tese (Doutorado) - Universidade Estadual do Norte Fluminense Darcy Ribeiro, Campos dos Goytacazes.

TIBOLA, C.S.; FACHINELLO, J.C.; GRÜTZMACHER, A.D.; PICALOTTO, L.; KRÜGER, L. Manejo de pragas e doenças na produção integrada e convencional de pêssegos. Revista Brasileira de Fruticultura, v.27, p.215-218, 2005.

WYATT, T.D. Method in studying insect behaviour. In: DENT, D.R.; WALTON, M.P. (Ed.). Method in ecological and agricultural entomology. New York: CAB Internacional, 1997. p.27-56.

Recebido em 27 de fevereiro de 2009 e aprovado em 29 de maio de 2009 